\title{
Weight discrimination within the healthcare system: Myth or reality?
}

\author{
Tracy S. Moreira-Lucas
}

\section{University of Toronto}

Obesity is a significant public health problem in Canada with approximately $24 \%$ of Canadians classified as obese. ${ }^{1}$ Obesity is associated with an increased risk for developing cardiovascular disease, type 2 diabetes and some cancers. ${ }^{1}$ The increased morbidity associated with obesity not only negatively impacts the patient, both physiologically and psychologically, but also places increased demands on the healthcare system. ${ }^{2}$ Despite their increased need for healthcare, obese individuals often face weight discrimination (WD), defined as the display of prejudicial judgment towards an individual based on their body weight. WD has been a topic of interest for over four decades but has only become increasingly important due to the rise in obesity prevalence. ${ }^{3}$

WD within the healthcare system may not be overt but according to researchers is a problem in need of attention. ${ }^{4-10}$ One of the earliest studies to examine physicians' attitudes towards obese patients was published in 1969 by Maddox and Liederman. ${ }^{3}$ In this study they showed that physicians $(n=491)$ practicing in the U.S. viewed their obese patients as unintelligent, unsuccessful, inactive and weak-willed. ${ }^{3}$ Similarly, $66 \%$ of general practitioners (GPs) ( $n=318$ ) in the U.S. reported that their obese patients lacked selfcontrol and $39 \%$ stated that their obese patients were lazy. ${ }^{4}$ These views were expressed despite the lack of evidence to support these beliefs. Another study of primary-care physicians $(n=620)$ showed that more than half viewed obese patients as awkward, unattractive and noncompliant. ${ }^{5}$ Also, more than half of the queried physicians stated that they viewed obesity as primarily a behavioural issue caused by physical inactivity and overeating, despite the wealth of evidence available to support that obesity is a multi-factorial condition that includes physiological and psychological components as well. ${ }^{1,5}$ The pervasive idea that obesity is simply caused by overeating and physical inactivity continues and is a belief that not only spans several health disciplines but many countries as well. One study conducted with GPs practicing in France $(n=600)$ found that $30 \%$ of them viewed overeating as the most important risk factor for obesity, ranked above genetic and environmental factors. ${ }^{6}$ Even physicians who specialize in treating obesity harbour anti-obesity beliefs/biases despite having knowledge of the multi-factorial nature of obesity. ${ }^{7}$ There is also evidence to suggest that the anti-obesity biases observed in some physicians are found in individuals studying to become physicians. ${ }^{8}$ In a study conducted on a sample of students enrolled in medical school, researchers found that the students characterized obese individuals as sloppy, lazy and lacking self-control. ${ }^{8}$ However, after receiving obesity-specific education, these negative attitudes towards obesity were shown to improve. ${ }^{8}$ This is promising because it shows that addressing these biases with further education, helps to decrease its pervasiveness, thus the key to thwarting WD may be in increasing education and awareness about the complex nature of the pathophysiology of obesity and its related conditions.

There are several questions that need to be answered in order to fully understand the effects WD has on obese patients. Are physicians failing to adequately treat their obese patients because of their negative feelings/ biases towards them? Do the perceived negative attitudes towards obese patients translate into poorer patient care? (i.e. are obese patients getting the appropriate treatment for their obesity) Are obese patients less likely to seek medical treatment because they feel their physicians lack an interest in caring for them? Do obese patients with unbiased doctors have better health outcomes then those seen by biased practitioners? One study reported that obese women are significantly less likely to undergo pelvic examinations compared to normal-weight women. ${ }^{9}$ Whether this was due to the patients' personal insecurities with their bodies or how they perceived their physicians 
c viewed them is unclear. Although there is little evidence to suggest that negative beliefs towards obesity translate into actions, there are concerns that they may and hence warrants further investigation. The concern regarding these anti-obesity biases is complex and multi-faceted which, if not addressed, may ultimately lead to poor patient care.

Discrimination against obese individuals amongst healthcare practitioners negatively impacts the health of obese patients. ${ }^{5,9,10}$ Educational conferences focusing on weight bias and stigma within the healthcare system are becoming increasingly available to healthcare practitioners and researchers but more work is needed to change these negative biases towards obesity. What we know is that WD is present in the healthcare system and that education which focuses on the pathogenesis of obesity, the associated conditions and most importantly the behavioural and psychological aspects leading to the condition can help to prevent WD. Furthermore, we know that the obesity epidemic continues on its upward climb, and healthcare practitioners will have to care for these patients and will have no choice but to find ways to accommodate them.

\section{References}

1. Shields $\mathrm{M}$, Carroll $\mathrm{MD}$, Ogden $\mathrm{Cl}$. Adult obesity prevalence in Canada and the United States. Natl Ctr Health Stats. 2011;56: 1-8.

2. Public Health Agency of Canada. Obesity in Canada- Health and economic implications. [Internet]. 2011 [cited 2014 Feb 10]. Available from: http:// www. phac-aspc.gc.ca/hp-ps/hl-mvs/oic-oac/econo-eng.php

3. Maddox GL, Liederman V. Overweight as a social disability with medical implications. J Med Educ. 1969;44: 214-20.

4. Foster GD, Wadden TA, Makris AP et al. Primary care physicians' attitudes about obesity and its treatment. Obes Res 2003;11:1168-1177.

5. Price JH, Desmond SM, Krol RA, Snyder FF, O'Connell JK. Family practice physicians' beliefs, attitudes, and practices regarding obesity. Am J Prev Med. 1987;3:339-45.

6. Bocquier A, Verger $\mathrm{P}$, Basdevant $\mathrm{A}$ et al. Overweight and obesity: knowledge, attitudes, and practices of general practitioners in France. Obes Res 2005;13:787-795.

7. Schwartz MB, Chambliss HO, Brownell KD, Blair SN, Billington C. Weight bias among health professionals specializing in obesity. Obes Res. 2003;11(9):1033-1039.

8. Wiese $\mathrm{HJ}$, Wilson JF, Jones RA, Neises M. Obesity stigma reduction in medical students. Int J Obes Relat Metab Disord. 1992;16:859-68.

9. Adams CH, Smith NJ, Wilbur DC, Grady KE. The relationship of obesity to the frequency of pelvic examinations: do physician and patient attitudes make a difference?. Women Health. 1993; 20:45-57.

10. Balkhi AM, Parent MC, Mayor M. Impact of Perceived weight discrimination on patient satisfaction and physician trust. Fat Studies. 2013; 2:45-55.

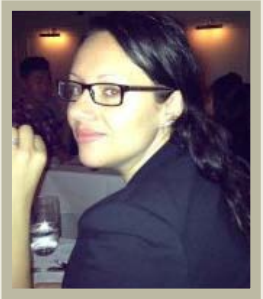

Tracy S. Moreira-Lucas

Tracy is a 3rd year PhD candidate in the Department of Nutritional Sciences at the University of Toronto. Her research interests lie in studying the role of nutrition in preventing chronic diseases, specifically type 2 diabetes. Her current research focuses on examining the effect of vitamin D supplementation on markers of diabetes. Tracy is passionate about teaching others about diabetes prevention and does this in her role as the vice-chair of the Canadian Diabetes Association University of Toronto Chapter (CDAUT). 